\title{
The Effect of a Full Bladder on Proportions of Diagnostic Ultrasound Studies in Children with Suspected Appendicitis
}

\author{
Marshall Ross, MD*; Sasha Selby"; Naveen Poonai, MD"; Helena Liu, BSc**; \\ Shabnam Minoosepehr, $\mathrm{MD}^{\dagger}$; Graham Boag, $\mathrm{MD}^{\ddagger}$; Robin Eccles, MD${ }^{\S}$; Graham Thompson, $\mathrm{MD}^{\dagger}$
}

\section{ABSTRACT}

Objectives: We examined the effect of a full bladder on proportions of diagnostic ultrasound (US) studies in children with suspected appendicitis. We also examined the effect of a full bladder on proportions of fully visualized ovaries on US in children with suspected appendicitis.

Methods: We conducted a retrospective health record review of children aged 2-17 years presenting to a tertiary pediatric emergency department (ED) with suspected appendicitis who had an ultrasound performed. We compared proportions of diagnostic US studies in children with full and sub-optimally filled bladders. We also compared proportions of ovarian visualization in females with full and sub-optimally filled bladders.

Results: 678 children were included in our final analysis. The proportion of diagnostic US studies did not vary significantly between groups with a full $(132 / 283,47 \%$, 95\% confidence interval [Cl] $38 \%-56 \%$ ) or sub-optimally filled bladder (205/ $395,52 \%, 95 \% \mathrm{Cl} 47 \%-57 \%)(p=0.17)$. Rates of ovarian visualization were higher in females with a full bladder (196/ $205,96 \%$, 95\% Cl 93\%-99\%) compared to those with a suboptimally filled bladder (180/223, 81\%, 95\% Cl 76\%-86\%) $(p<0.01)$.

Conclusions: Administrators and clinical decision makers should consider removing routine bladder filling practice from current pediatric appendicitis protocols in males and in pre-pubertal females where ovarian pathology is not suspected. Selective bladder filling prior to US should be performed in females when ovarian pathology is suspected.

\section{RÉSUMÉ}

Objectifs: L'étude visait à examiner, d'une part, l'effet d'une vessie pleine sur la proportion d'examens de diagnostic par échographie effectués chez des enfants souffrant d'une appendicite présumée et, d'autre part, l'effet d'une vessie pleine sur la proportion de visualisation complète des ovaires à l'échographie chez les filles souffrant d'une appendicite présumée.

Méthode: Les auteurs ont mené un examen rétrospectif de dossiers médicaux d'enfants âgés de 2 à 17 ans, qui sont allés au service des urgences pédiatriques de soins tertiaires pour une appendicite présumée et qui ont été soumis à une échographie. Ont été comparées la proportion d'enfants ayant subi une échographie de diagnostic, la vessie pleine, avec celle d'enfants ayant subi le même examen, la vessie moins que pleine. Une comparaison a aussi été établie entre la proportion d'ovaires visualisés chez les filles ayant une vessie pleine et celle d'ovaires visualisés chez les filles ayant une vessie moins que pleine.

Résultats: L'analyse a finalement porté sur 678 dossiers d'enfant. La proportion d'examens de diagnostic par échographie était sensiblement la même dans les deux groupe d'enfants, soit celui ayant la vessie pleine (132/283; $47 \%$; intervalle de confiance à $95 \%$ [IC] : 38-56\%) et celui ayant la vessie moins que pleine (205/395; $52 \%$; IC à $95 \%$ : 47-57\%) ( $p=0,17)$. Le taux de visualisation des ovaires était plus élevé chez les filles ayant la vessie pleine (196/205; $96 \%$; IC à $95 \%$ : 93-99\%) que chez les filles ayant la vessie moins que pleine (180/223; $81 \%$; IC à $95 \%: 76-86 \%)(p<0,01)$.

Conclusions: Les gestionnaires et les décideurs en matière de pratiques cliniques devraient envisager le retrait du remplissage systématique de la vessie, des protocoles actuels de traitement de l'appendicite, chez les garçons et chez les filles prépubères ainsi que chez les filles chez qui tout doute d'affection des ovaires est écarté. Par contre, on devrait procéder à un remplissage sélectif de la vessie avant une échographie chez les filles chez qui il y a des doutes quant à I'existence d'une affection des ovaires.

Keywords: Appendicitis, ultrasound, diagnosis, pediatrics

From the *Department of Emergency Medicine, †Pediatric Emergency Medicine, ¥Department of Radiology, and the $\S$ Department of Pediatric Surgery, University of Calgary, Calgary, AB; đMedical Undergraduate at University of Limerick, Limerick, Ireland; ॥Department of Pediatrics, Western University, London, ON; and **Department of Medical Education, University of Alberta, Edmonton, AB.

Correspondence to: Graham Thompson, Email: Graham.Thompson@albertahealthservices.ca 


\section{INTRODUCTION}

Appendicitis is the most common non-traumatic surgical emergency in children. ${ }^{1,2}$ Despite the high incidence of appendicitis, the diagnosis remains challenging, as patients often lack the classic historical and physical examination findings. ${ }^{3}$ This has led to substantial reliance on diagnostic imaging, ${ }^{4}$ including ultrasound (US), computed tomography (CT), and staged protocols. ${ }^{5,6}$ Due to increased availability of US and increased awareness of potential adverse outcomes associated with ionizing radiation, particularly in young children, ${ }^{7}$ many health care providers choose ultrasonography as their first line imaging modality to diagnose appendicitis in the pediatric population. ${ }^{8}$

The diagnostic accuracy of ultrasonography is subject to visualization of the structures to be assessed, which in the case of appendicitis is often limited. ${ }^{9}$ In an attempt to optimize visualization of pelvic and intraabdominal structures, some institutions require bladder filling prior to pelvic US examination for suspected appendicitis. This can be uncomfortable for patients and leads to delays in test acquisition and subsequent diagnosis. ${ }^{10}$ While there is some evidence that delays in diagnosis and treatment make little difference to clinically important outcomes, ${ }^{10-13}$ other studies suggest treatment delays may lead to increased rates of perforation. ${ }^{14}$

Our objective was to determine the value of bladder filling prior to pelvic US in children with suspected appendicitis. Specifically we examined the impact of a full versus sub-optimally filled bladder on the proportions of diagnostic pelvic US studies in males and females as well as proportions of fully visualized ovaries.

\section{METHODS}

\section{Study design}

We performed a retrospective health record review, combining data that were available from a study previously reported by our team ${ }^{9}$ with US images to calculate bladder size. This study was approved by the Conjoint Health Research Ethics Board of the University of Calgary.

\section{Study setting and population}

This study was conducted in the departments of Pediatric Diagnostic Imaging and Pediatric Emergency
Medicine at the Alberta Children's Hospital (ACH). $\mathrm{ACH}$ is the pediatric tertiary care facility serving southern Alberta, western Saskatchewan and eastern British Columbia, with an annual emergency department (ED) census of approximately 72,000 visits and a catchment area of 1.8 million.

Diagnostic imaging studies were reviewed for children aged 2-17 years who received an US for suspected appendicitis during the predefined inclusive time period of January 1, 2007, through December 31, 2008. Eligible subjects were identified using electronic patient tracking databases hosted by the department of radiology. Databases were searched for records of children receiving an US with the "US Appendix" protocol code. US studies were performed by trained US technicians and reported by staff radiologists. At our pediatric center, US imaging of the pelvis is limited to the transabdominal approach. Therefore, no transvaginal USs were evaluated.

\section{Study protocol}

Baseline demographic and clinical data were available from our previously published health record review, ${ }^{9}$ in which we collected demographic data, ED visit details, results of laboratory investigations, surgical considerations, frequency of return visits to the $\mathrm{ED}$, and imaging reports. These data were used in a previous study ${ }^{9}$ to examine the outcomes of children with suspected appendicitis after US examination failed to identify the appendix. In particular, we studied children's outcomes, including their disposition, the proportion returning for care, the proportion undergoing surgical intervention, and the number of "missed" appendicitis cases, as well as the test characteristics of secondary signs of inflammation in this population. For the purpose of this study the existing database was expanded by reviewing all available US images and diagnostic imaging reports. One of our authors (S.S.) received standardized training in research ethics, methodologies, and data entry, and collected additional data for all US images available on PACS (Picture Archiving and Communications System), including bladder shape, bladder dimensions, appendix visualization, ovarian visualization, and alternative diagnoses using a standardized data collection form. Radiology reports were reviewed to assess for ovarian visualization. Predictor and outcome variables were operationalized as much as possible to reduce the potential for bias. 


\section{Definitions}

An "incompletely visualized appendix" was defined as any US study that failed to visualize the entire appendix. A "normal" US fully visualized a normal appendix in males or a normal appendix and ovaries in females and found no alternate diagnosis. An "alternative diagnosis" was defined as a finding on US that was considered the primary diagnosis on the ED record other than appendicitis. A “diagnostic US" included: a normal US, was diagnostic of appendicitis, or made an alternative diagnosis. All scans that did not meet diagnostic US criteria were classified as non-diagnostic. We considered patients to have a full bladder if the percent of expected bladder capacity (PEBC) was greater than or equal to $75 \%$. Those with PEBC less than $75 \%$ were considered to have sub-optimally filled bladders. We considered a radiology report that included a term such as "ovaries are fully visualized" as positive for ovarian visualization. We did not directly collect data on full adnexal visualization but collected data from positive adnexal findings under "alternative diagnosis."

\section{Estimating bladder size, age-based normal and bladder fullness}

In order to estimate bladder size on US we collected data on anterior-posterior diameter (APd) and the width $(\mathrm{W})$ of the bladder from the transverse view. The bladder cranio-caudal diameter (CCd) was determined from the sagittal view. Accurate estimation of bladder volume must take into consideration the bladder shape. ${ }^{15,16}$ As outlined in detail by Kuzmic et al. ${ }^{16}$ we classified bladder shape as round, ellipsoid, cuboid, triangular, or undefined. Estimated bladder volume (EBV) was then calculated with the following equation:

$$
\mathrm{BV}=\mathrm{W} \times \mathrm{APd} \times \mathrm{CCd} \times \mathrm{k}
$$

where $\mathrm{k}$ varied with bladder shape. Specifically, the $\mathrm{k}$ values used were 0.561 for round, 0.923 for cuboid, 0.802 for ellipsoid, 0.623 for triangular, and 0.749 for undefined, as recommended by Kuzmic et al. ${ }^{16}$

Estimated bladder capacity (EBC) was defined using the following validated age-based equation as originally presented by Kaefer et al.: ${ }^{17}$

$$
\mathrm{EBC}(\text { in } \mathrm{mL})=133.08 \times \text { Age }^{0.4}
$$

We then calculated the percent of expected bladder capacity (PEBC) expressed as a percentage by dividing the EBV by the EBC as follows:

$$
\mathrm{PEBC}=\mathrm{EBV} / \mathrm{EBC} \times 100 \%
$$

All PEBC greater than $100 \%$ are reported as $100 \%$.

\section{Calculating the effect of bladder fullness on the rate of diagnostic US}

The primary outcome variable was the proportion of diagnostic USs. We hypothesized that the group with sub-optimally filled bladders would be non-inferior to the group with full bladders. We defined a priori subgroup analyses based on sex for the primary outcome variable in groups with both full and sub-optimally filled bladders. The secondary outcome variable was the proportion of complete ovarian visualization in females.

We compared proportions of diagnostic USs in patients with filled versus sub-optimally filled bladders using statistical analysis often used in non-inferiority trials. Given the retrospective nature of our study we, by definition, did not perform a formal non-inferiority trial. Rather, we utilized a statistical technique commonly employed in non-inferiority trials to examine for a clinically significant difference or lack thereof in our primary outcome measure. Specifically, we calculated the difference in mean proportions of diagnostic USs in those with full and sub-optimally filled bladders. We considered a $10 \%$ difference in mean proportions of diagnostic USs to be clinically significant and set this as our delta value. ${ }^{18}$ In other words, we considered the performance of US with sub-optimally filled bladders to be non-inferior to US with full bladders if the mean proportion of diagnostic USs was no lower than $10 \%$ compared to the mean proportions in those with full bladders. The same strategy was used in our sub-group analysis by sex as well as to assess ovarian visualization.

\section{RESULTS}

Of 978 records reviewed in our original study, ${ }^{9}$ we were able to obtain sufficient images to calculate bladder size in 678 patients. In 55 cases, the US images were insufficient in areas scanned to estimate bladder size. In 245 cases, we were unable to obtain any images from the diagnostic imaging database. Patient identification numbers from our original study often had written US reports available but no cached images in our diagnostic 
imaging database. We were therefore unable to calculate bladder size and include these studies in our final analysis. However, no statistical demographic differences were found between those included and not included in the study (Appendix 1). A flow diagram of reviewed charts appears in Figure 1.

Demographic parameters and US classifications are presented in Table 1. The proportion of diagnostic US studies did not vary significantly between groups with a full or sub-optimally filled bladder (47\% [132/283] vs. $52 \%$ [205/395] respectively, $p=0.17)$. This observation held true in both male and female cohorts $(p=0.24$ and $p=0.58$ respectively). Rates of ovarian visualization were higher in females with a full bladder compared to those with a sub-optimally filled bladder (96\% [196/205] vs. $81 \%$ [180/223] respectively, $p<0.01)$. There was a greater proportion of males with sub-optimally filled bladders as compared to females $(69 \%$ [172/250] vs. $52 \%$ [223/428] respectively, $p<0.01)$. Further sub-classifications of diagnostic USs are reported in Table 2.

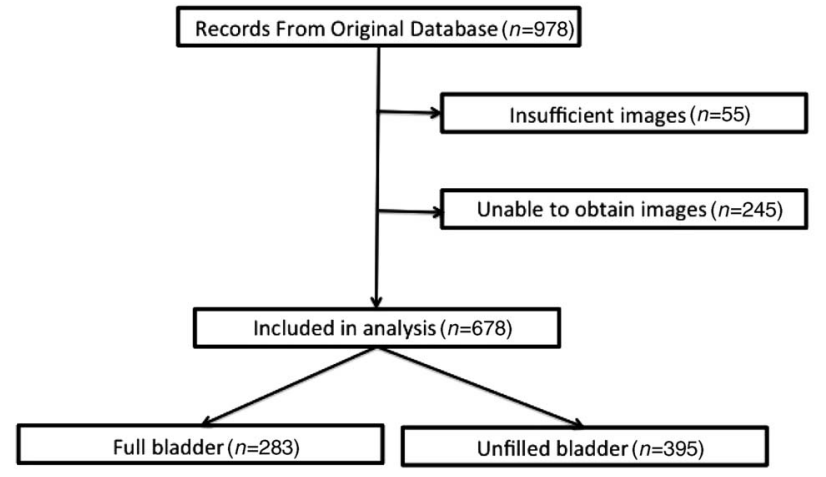

Figure 1. Patients included in final analysis
Results of the primary outcome are displayed graphically in Figure 2. The group with sub-optimally filled bladders was non-inferior to the group with full bladders. This finding was true in both our male and female subgroups. The group with unfilled bladders had significantly lower rates of ovarian visualization than those with full bladders. However, it is plausible that the true treatment difference is less than our clinically relevant delta value of $10 \% .^{19}$

\section{DISCUSSION}

In this study, we examined the relationship between bladder volume and a diagnostic US examination in children with suspected appendicitis. Our data demonstrate that the proportion of diagnostic US studies does not vary significantly with a full or sub-optimally filled bladder and this observation held true across sex.

These results suggest that omitting routine bladder filling from diagnostic protocols for suspected appendicitis may not change diagnostic accuracy. Omitting routine bladder filling could improve emergency department flow, prevent patients from enduring a painful full bladder, and decrease the need for invasive procedures at institutions that fill bladders using catheterization. While the American Institute of Ultrasound in Medicine still recommends bladder filling for pelvic ultrasonography in their 2010 guidelines, ${ }^{20}$ other experts in the field have cogently argued that this practice may be unnecessary with modern US technology, ${ }^{21}$ albeit in an older population. Consideration should be made for discontinuing the

\begin{tabular}{|c|c|c|c|c|}
\hline & & Full bladder & Unfilled bladder & $p$ value* \\
\hline \multirow[t]{5}{*}{$N$} & All & 283 & 395 & \\
\hline & Female & $205(73.5 \%)$ & $223(56.5 \%)$ & \\
\hline & Male & $78(27.6 \%)$ & $172(43.5 \%)$ & \\
\hline & Age (SD) & $12.8(3.5)$ & $11.0(4.0)$ & 0.99 \\
\hline & CTAS, mean (SD) & $3.2(0.4)$ & $3.0(0.5)$ & 0.99 \\
\hline \multirow[t]{3}{*}{ Diagnostic } & All & $132(47 \%)$ & $205(52 \%)$ & 0.17 \\
\hline & Female & $85(41 \%)$ & $95(42 \%)$ & 0.24 \\
\hline & Male & $47(60 \%)$ & $110(64 \%)$ & 0.58 \\
\hline \multicolumn{2}{|c|}{ Ovarian visualization (females only) } & $196(96 \%)$ & $180(81 \%)$ & $<0.01$ \\
\hline \multicolumn{5}{|c|}{$\begin{array}{l}\text { Odds ratios report the odds of having a non-visualized scan with an unfilled bladder. } \\
\text { CTAS: Canadian Triage Assessment Score } \\
\text { 95\% Cl: } 95 \% \text { confidence interval } \\
\text { SD: standard deviation } \\
\text { *Z-tests were used to calculate } p \text { values }\end{array}$} \\
\hline
\end{tabular}




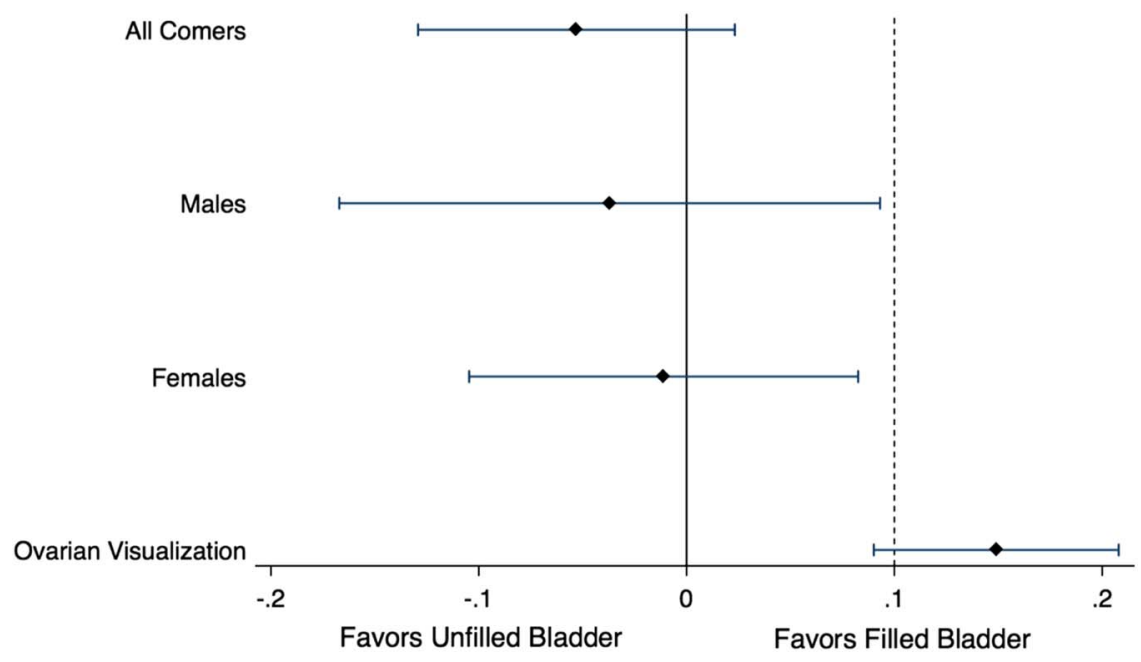

Figure 2. Non-inferiority Analysis

Delta of $10 \%$ represented with dashed line

\begin{tabular}{|c|c|c|c|c|c|c|}
\hline & & \multirow[b]{2}{*}{$n$} & \multirow[b]{2}{*}{ Diagnostic } & \multicolumn{3}{|c|}{ Diagnostic } \\
\hline & & & & Normal & Appendicitis & Alternative Diagnosis \\
\hline \multirow[t]{3}{*}{ Full bladder } & All & 283 & $132(47 \%)$ & $36(13 \%)$ & $68(24 \%)$ & $28(10 \%)$ \\
\hline & Female & 205 & $85(41 \%)$ & $20(10 \%)$ & $40(20 \%)$ & $25(12 \%)$ \\
\hline & Male & 78 & $47(60 \%)$ & $16(21 \%)$ & $28(36 \%)$ & $3(4 \%)$ \\
\hline \multirow[t]{3}{*}{ Bladder unfilled } & All & 395 & $205(52 \%)$ & $54(14 \%)$ & $124(31 \%)$ & $27(7 \%)$ \\
\hline & Female & 223 & $95(43 \%)$ & $28(13 \%)$ & $51(23 \%)$ & $16(7 \%)$ \\
\hline & Male & 172 & $110(64 \%)$ & $26(15 \%)$ & $73(42 \%)$ & $11(6 \%)$ \\
\hline
\end{tabular}

practice of routine bladder filling in pediatric patients with suspected appendicitis.

In contrast to the appendix, we found that full ovarian visualization was significantly higher in those with full bladders as compared to those with unfilled bladders. This is likely due to the acoustic window created by a fluid filled structure. Therefore, it is reasonable to consider bladder filling in female patients presenting with abdominal and/or pelvic pain in whom appendicitis and ovarian pathology are in the differential diagnosis (i.e., post-pubertal females). Further study is required to delineate the value of ovarian visualization in guiding management. Our data demonstrate that if ovarian pathology is not suspected, bladder filling may not be routinely required prior to US examination. In collaboration with radiology departments, emergency physicians should consider implementing protocols that do not require bladder filling in male patients and female patients with no clinical concern for ovarian pathology. This may speed throughput and improve patient satisfaction. However, these process measures and patient-reported outcome measures, such as time to US and patient comfort, were not available in this study.

The higher proportion of males with unfilled bladders was an unexpected finding. It may be that the bladder filling protocol was less strictly adhered to in the male population due to absence of clinical concern for pathology in pelvic organs.

\section{LIMITATIONS}

Our study faces limitations due to incomplete and missing images. This is unlikely to significantly bias our results, given similarities between groups with and without available complete images, as described in Appendix 1.

Our definition of a full bladder being greater than $75 \%$ of the PEBC is arbitrary. However, this value was felt to be clinically appropriate and a change in the cutoff would be unlikely to change our results. 
Likewise our delta value of $10 \%$ for non-inferiority is arbitrary but is clinically reasonable.

Our statistical analysis was performed on retrospective data available from a previous trial. As such this study is not a non-inferiority trial but rather a retrospective analysis using non-inferiority statistical techniques to analyze available data.

Other limitations include our inability to fully characterize and adjust for other patient factors that may have limited diagnostic accuracy, such as patient cooperation, position of the appendix, such as retrocecal, and body habitus, as these data were not available. Classification of bladder shapes and bladder measurements was performed by a single author, and so we were unable to calculate an interclass correlation coefficient, and significant error may have occurred.

\section{CONCLUSION}

The presence of a full bladder prior to US for suspected appendicitis was not associated with higher proportions of diagnostic USs for pediatric appendicitis in our population. However, the proportion of USs with fully visualized ovaries was significantly higher when the bladder was full. Administrators and clinical decision makers should consider omitting routine bladder filling from current pediatric appendicitis protocols in males and in pre-pubertal females where ovarian pathology is not suspected. Selective bladder filling prior to US should be performed in females when ovarian pathology is suspected. Further studies are required to fully delineate the role of a full bladder in US studies for suspected pediatric appendicitis.

Competing Interests: None declared.

\section{SUPPLEMENTARY MATERIAL}

To view supplementary material for this article, please visit http://dx.doi.org/10.1017/cem.2016.23

\section{REFERENCES}

1. Reynolds SL, Jaffe DM. Diagnosing abdominal pain in the pediatric emergency department. Pediatr Emerg Care 1992; 8(3):126-8.

2. Scholer SJ, Pituch K, Orr DP, et al. Clinical outcomes of children with acute abdominal pain. Pediatrics 1996; 98(4 Pt 1):680-5.

3. Becker T, Kharbana A, Bachur R. Atypical clinical features of pediatric appendicitis. Acad Emerg Med 2007;14(2):124-9.
4. Pines JM. Trends in the rates of radiography use and important diagnoses in emergency department patients with abdominal pain. Med Care 2009;47(7):782-6.

5. Krishnamoorthi R, Ramarajan N, Wang NE, et al. Effectiveness of a staged US and CT protocol for the diagnosis of pediatric appendicitis: reducing radiation exposure in the age of ALARA. Radiology 2011;259(1):231-9.

6. Ramarajan N, Krishnamoorthi R, Barth R, et al. An interdisciplinary initiative to reduce radiation exposure: evaluation of appendicitis in a pediatric emergency department with clinical assessment supported by a staged ultrasound and computed tomography pathway. Acad Emerg Med 2009;16(11):1258-65.

7. Brenner D, Elliston C, Hall E, et al. Estimated risks of radiation-induced fatal cancer from pediatric CT. AfR Am $\mathcal{7}$ Roentgenol 2001;176(2):289-96.

8. Thompson GC, Schuh S, Gravel J, et al. Variation in the diagnosis and management of appendicitis at Canadian pediatric hospitals. Acad Emerg Med 2015;22(7):811-22.

9. Ross MJ, Liu H, Netherton SJ, et al. Outcomes of children with suspected appendicitis and incompletely visualized appendix on ultrasound. Acad Emerg Med 2014;21(5): 538-42.

10. Poonai N, Gregory J, Thompson G, et al. Is pelvic ultrasound associated with an increased time to appendectomy in pediatric appendicitis? 7 Emerg Med 2014;47(1):51-8.

11. Abou-Nukta F, Bakhos C, Arroyo K, et al. Effects of delaying appendectomy for acute appendicitis for 12 to 24 hours. Arch Surg 2006;141(5):504-7.

12. Yardeni D, Hirschi RB, Drongowski RA, et al. Delayed versus immediate surgery in acute appendicitis: do we need to operate during the night? 7 Pediatr Surg 2004; 39(3):464-9.

13. Taylor M, Emil S, Nguyen N, et al. Emergent vs urgent appendectomy in children: a study of outcomes. 7 Pediatr Surg 2005;40(12):1912-5.

14. Papandria D, Goldstein SD, Rhee D, et al. Risk of perforation increases with delay in recognition and surgery for acute appendicitis. 7 Surg Res 2013;184(2):723-9.

15. Bih LI, Ho CC, Tsai SJ, et al. Bladder shape impact on the accuracy of ultrasonic estimation of bladder volume. Arch Phys Med Rebabil 1998;79(12):1553-6.

16. Kuzmić AC, Brkljacić B, Ivanković D. Bladder shape impact on the ultrasonic measurement of bladder volume in children. Pediatr Radiol 2003;33(8):530-4.

17. Kaeffer M, Zurakowski D, Bauer SB, et al. Estimating normal bladder capacity in children. $\mathcal{F}$ Urol 1997;156(6):2261-4.

18. Wiens BL. Choosing an equivalence limit for noninferiority or equivalence studies. Control Clin Trials 2002;23(1):2-14.

19. Piaggio G, Elbourne DR, Altman DG, et al. Reporting of noninferiority and equivalence randomized trials: an extension of the CONSORT statement. FAMA 2006; 295(10):1152-60.

20. American Institute of Ultrasound in Medicine. AIUM practice guideline for the performance of pelvic ultrasound examinations. 7 Ultrasound Med 2010;29(1):167-72.

21. Benacerraf B. Filling of the bladder for pelvic sonograms: an ancient form of torture. 7 Ultrasound Med 2003;22(3): 239-41. 